\title{
On Practice of the Effect of Systematic Family Nursing Guidance on the Quality of Life of Elderly Patients with Implanted Cardiac Pacemaker
}

\author{
Xin Ao \\ Nursing Department of Medical School, Yangtze University, Jingzhou, China \\ Email: cjdxhlx@126.com
}

How to cite this paper: Ao, X. (2018) On Practice of the Effect of Systematic Family Nursing Guidance on the Quality of Life of Elderly Patients with Implanted Cardiac Pacemaker. Yangtze Medicine, 2, 171-177. https://doi.org/10.4236/ym.2018.23018

Received: June 10, 2018

Accepted: August 27, 2018

Published: August 30, 2018

Copyright $\odot 2018$ by author and Scientific Research Publishing Inc. This work is licensed under the Creative Commons Attribution International License (CC BY 4.0).

http://creativecommons.org/licenses/by/4.0/ cC) (i) Open Access

\begin{abstract}
Objective: To inquire into the effect of systematic family nursing guidance on improving the Quality of Life for elderly patients with implanted cardiac pacemaker. Methods: 41 elderly patients in accordance with the corresponding requirements were studied after offering systematic family nursing guidance and surveyed through the questionnaires about the QOL (quality of life) respectively 1 month, 3 months and 6 months after leaving hospital. Results: Compared with that of 1 month and 3 months after the operation, the questionnaire scores for 6 months after the operation were found to have significant improvement, and the differences were statistically significant. Conclusion: Systematic family nursing guidance can effectively improve the QOL for elderly patients with implanted cardiac pacemaker, which makes them spend their old age in comfort and happiness.
\end{abstract}

\section{Keywords}

Cardiac Pacemaker, Elderly Patients, Family Nursing Guidance, Quality of Life, SF-36 Health Survey

\section{Introduction}

With the growing incidence of heart pace-making and conductance dysfunction in recent years, there is an increase for the cases of cardiac permanent pacemaker implanting [1], and the elderly patients have become the major groups of cardiac pacing therapy in China at present. According to the surveys, implanting cardiac permanent pace-makers have some negative effects on the patients' qual- 
ity of life [2] [3]. Especially among the elderly patients, due to their falling of physiological function and of behavioral competence, such problems were found after implanting cardiac pacemakers as carrying a heavy physiological burden, being severely lack of self-care and substantially reducing social activities of connections, even though a small number of patients place a heavy burden on their family life. To inquire into it, 41 elderly patients with implanted cardiac pacemakers were offered systematic family nursing guidance and their quality of life was followed up. The details were as below.

\section{Materials and Methods}

\subsection{Materials}

According to the new standard age division of WHO (World Health Organization), those people who are between 60 and 74 years old are defined as quasi elderly people, and those over 75 years old are elderly ones. So that 41 patients with implanted cardiac permanent pacemakers (male, 25; female, 16), whose age ranging from 60 to 82 years old, were chosen as the study objects, and their average age was $71 \pm 0.926$ years old. All of them are informed consent patients willing to receive the nursing guidance, excluding those who are not supported by their families or those who have lost their ability to learn. The types of the pacemakers used as: 1) dual chamber pacemaking, dual chamber apperceive and dual mode of triggering or inhibiting (DDD) for 6 patients; 2) ventricular Pacing, ventricular perception and then demonstrate an inhibitory reaction (VVI) for 21 patients; 3) atrial pacing, atrial perception and lead to an inhibitory reaction (AAI) for 7 patients; 4) dual chamber pacemaking, dual chamber perception, dual responses of triggering or inhibiting and the heart rate of the patient can be accommodated (DDDR) for 2 patients; 5) ventricular pacemaking, ventricular perception, mode of inhibition, and con adapt to the patient's heart rate changes (VVIR) for 5 patients. All of the patients are the elderly people living in the same community with the same regional cultural backgrounds, including the same dialect, the same living environment, the same customs and communication condition, and the same folk beliefs and economical environment, which can make sure the comprehension, the implementation and the compliance of the patients are more similar with regard to the systematic family nursing guidance.

\subsection{Methods}

All the following methods of nursing guidance do not violate ethics or morality, without any harm to the patients.

\subsubsection{Family Nursing Guidance Method}

When leaving the hospital, all the elderly patients were offered a full evaluation of their psychology, physiology and family support, then make systematic family nursing guidance, create health records and designate special persons to take charge of guidance and management. After being discharged from hospital, the regular follow-ups were made by telephone and through home visit according to 
the family nursing guidance: in general, within 1 month will have twice home visit and telephone follow-up once during one or two days; within 3 months will have home visit once a month, telephone follow-up once during one or two weeks; within 6 months will have home visit twice, telephone follow-up once a month, if necessary, the home visit and telephone follow-up can be added more times. Furthermore, during the process, the new things that may occur or hare existed had to be concerned and evaluated so that targeted guidance could be provided. The following is details:

1) Psychological guidance

The majority of the patients leaving hospital were evaluated to show such mental stress as worry, concern, intension and even anxiety, which lead the patient to feel restless, have difficulty in falling asleep, do not dare to raise arms, fear doing exercise and be too cautious to handle things, which finally will have strongly negative impact on the quality of life [4]. Therefore, the active psychological counseling was adopted to solve these problems: firstly, listen attentively to the patients' catharsis of negative emotions and let the patients describe the details of their emotion, then accurately find the cause of the psychological stress, finally guide the patients to reduce and eliminate those factors. For instance, to help the patients objectively analyze the odds of accidents after implanting the pacemakers without exaggeration, cope patiently with each of their doubts, explain the reasons why palpitation, dizziness and malaise occur when the pacemakers work, introduce such knowledge as the service life, the time for battery depletion and change of pacemakers to patients to alter their negative perceptions of pacemakers. After that, the patients are encouraged to do some things that can adjust emotions and attitudes with the accompanying of family members and nurses. For example: being involved in social activities; cultivating such hobbies as reading books or newspapers, painting, playing chess, weaving, listening to music, singing, dancing, riding a bicycle or playing Taiji, visiting friends or relatives to enjoy affection. Thus, through eliminating the patients' doubts, their attention can be distracted, then they can actively pursue their happiness so much to fade away the negative effects of pacemakers, patients can also be more confident and in a positive frame of mind to face life, which shortens their psychological adaptation process.

2) Behavior guidance

Behavior guidance including the following 7 aspects: 1) Guide the patients to make a medical card with the name, the diagnosis, the type of the pacemakers, the date of implantation, the address, the telephone number, contacting hospital name and the primary nurse. They are required to carry the card with themselves so that they can receive help timely when some unexpected condition occurs. 2) Guide the patients to design self- monitoring record including date, time, pulse, hematopiesis, adverse effects, the time for taking medicine, then generated all information into a table. 3) Guide the patients to learn to feel pulse, measure hematopiesis and make a correct monitoring record. 4) Instruct the patients to exercise the intraoperative limb, gradually increase the intensity and the 
range of intraoperative limb activities, avoid taking repeatedly violently actions such as swinging arms and expanding chest and striking the operated-side limb. 5) Instruct the patients to use pacemakers correctly, steering away from high-voltage electric field and magnetic field, also moving away from the environment of strong magnetic field; not to apply electrotherapy, magnetic therapy and magnetic resonance, to make their mobile phones away from the pacemakers, but have access to household appliances and take an elevator, an aircraft and an electric vehicle. Avoid heavier physical labor and excessive weight on the shoulder; keep the skin dry and clean where a pacemaker is implanted, wear soft underwear, and prevent patients from scratching to avoid infection when facing itchy skins. 6) Instruct the patients to make possible arrangement for their daily life to combine rest, entertainment with housework, record the monitoring sheet regularly, take medicine regularly, take food on time and choose the highly digestible foods rich in vitamins and fiber. 7) Guide the patients to master self-protective methods and simple emergency measures like: heart palpitation, chest distress and lack of strength etc., the patients should stop all activities to have a bed rest, and then contact the hospital by telephone after the symptom is alleviated, finally go to the hospital if necessary.

3) Family Members' Guidance

Family members were introduced the knowledge related to precautions and rehabilitation training after implanting the pacemakers in the patients so that they can offer comprehensive monitoring to the patients in life. Instruct the family members accompany the patients come to hospital to follow up within the time prescribed; usually communicate with the family members of the patients and encourage them to look after the patients, visit or accompany the elderly so that they can feel family warmth and affection, experience the joy of life and ignite their confidence in life.

\subsubsection{Evaluation Method}

The SF-36 scale (Chinese version) was adopted [5] [6] to follow up inquiry into the patients' quality of life, it yields an 8-scale profile of functional health and well-being scores as well as psychometrically-based physical and mental health summary measures: Physiological Function (PF), Role-physical (RP), Bodily Pain (BP), Vitality (VT), Social Function (SF), Role Emotional (RE) and Mental Health $(\mathrm{MH})$. There are total 36 items. And the eight scaled scores are the weighed sums of the questions in this section. Each scale is then directly transformed into a 0 - 100 scale on the assumption that each question carries equal weight. So the higher some patient's scores is, the higher his quality of life is.

\subsubsection{Statistical Methods}

All the data collected were dealt through the software-SPSS11.5. Dimensional scores are expressed by means of $(\bar{x})$ plus or minus standard deviation $( \pm S)$. The results of measurements in different time but the same dimension are compared between groups by nonparametric tests. 


\section{Results}

The patients' quality of life has increasingly improved after the system family nursing guidance, 3 months after surgery, the scores of quality of life were higher than that of 1 month after surgery, 6 months after surgery, the scores of quality of life were higher than that of 3 months after surgery (Table 1). The nonparametric comparison were carried out on the three group score, and the results have significant differences: between 1 month and 3 months postoperative $(P=$ 0.001 and $\mathrm{P}=0.000)$, between 3 month and 6 months after operation $(\mathrm{P}=$ $0.000)$, and between 1 month and 6 months after surgery $(P=0.000)$, which were also statistically significant (Table 2), showing that system family nursing guidance were effective in improving the patients' quality of life with implanted pacemakers.

\section{Results Analysis}

Systematic nursing is an advanced nursing method proposed with the change of medical model. It emphasizes that nursing is to help patients solve health

Table 1. Evaluation of scores on quality of life for patients guided by systematic family care $(\bar{x} \pm \mathrm{S}, \mathrm{n}=41)$.

\begin{tabular}{|c|c|c|c|}
\hline team & 1 month & 3 months & 6 months \\
\hline Physical Function (PF) & $70 \pm 3.604$ & $75 \pm 4.100$ & $84 \pm 2.824$ \\
\hline Role Physical (RP) & $54 \pm 0.958$ & $66 \pm 1.769$ & $86 \pm 3.515$ \\
\hline Bodily pain (BP) & $77 \pm 2.461$ & $84 \pm 2.957$ & $92 \pm 2.292$ \\
\hline General Health (GH) & $72 \pm 1.933$ & $75 \pm 2.186$ & $84 \pm 1.651$ \\
\hline Vitality (VT) & $60 \pm 2.586$ & $71 \pm 3.767$ & $83 \pm 2.488$ \\
\hline Social Function (SF) & $51 \pm 0.821$ & $69 \pm 1.967$ & $80 \pm 1.745$ \\
\hline Role Emotional (RE) & $60 \pm 1.440$ & $71 \pm 1.376$ & $85 \pm 1.730$ \\
\hline Mental Health (MH) & $49 \pm 0.790$ & $67 \pm 1.491$ & $85 \pm 2.409$ \\
\hline
\end{tabular}

Table 2. Comparison of patients' quality of life scores between the three groups.

\begin{tabular}{ccccccc}
\hline & \multicolumn{2}{c}{1 and 3 months } & \multicolumn{2}{c}{3 and 6 months } & 1 and 6 months \\
\cline { 2 - 7 } team & $\mathrm{Z}$ & $\mathrm{P}$ & $\mathrm{Z}$ & $\mathrm{P}$ & $\mathrm{Z}$ & $\mathrm{P}$ \\
\hline Physical Function (PF) & -3.742 & 0.001 & -5.749 & 0.000 & -9.491 & 0.000 \\
Role Physical (RP) & -5.207 & 0.000 & -5.207 & 0.000 & -10.414 & 0.000 \\
Bodily pain (BP) & -5.207 & 0.000 & -5.207 & 0.000 & -10.414 & 0.000 \\
General Health (GH) & -3.897 & 0.000 & -5.862 & 0.000 & -9.759 & 0.000 \\
Vitality (VT) & -5.114 & 0.000 & -5.254 & 0.000 & -10.368 & 0.000 \\
Social Function (SF) & -5.208 & 0.000 & -5.208 & 0.000 & -10.416 & 0.000 \\
Role Emotional (RE) & -5.207 & 0.000 & -5.207 & 0.000 & -10.414 & 0.000 \\
Mental Health (MH) & -5.207 & 0.000 & -5.207 & 0.000 & -10.415 & 0.000 \\
\hline
\end{tabular}

Comments: Z (Standardized Test Statistic); P (adj. sig). 
problems from the aspects of physical, psychological and social support, which are more scientific, systematic and complete than the traditional nursing method. For this reason, by uses the characteristics of systemic care, this study applied the perspectives and methods of systemic care to family nursing, to implement a planned, purposeful, and step-by-step approach to patients with pacemakers returning home, thus to improve the effectiveness of family nursing guidance.

It was showed as the result that the major problems the patients suffered from within 1 month after operation were mental disorder, weakened physiological and declining social function, and all the scores were lower in those three scales, which may be closely related to the physiological and psychological characteristics as well as pacemakers' working: firstly, the elderly patients' cognitive hypofunction, with the implantation and working of pacemakers, they became more dependent upon the pacemakers, cared about and even worried about them all the time; secondly, the increase of sensitivity for the elderly patients lead them to mistake the palpitation resulting from competitive cardiac rhythm and the discomfort arising from discharge for dysfunction of pacemakers themselves; thirdly, the implanted pacemakers among the elderly patients were usually single-chamber cardiac ones, often with such syndrome as dizziness, fullness in head breath hard and lacking in strength, which increased the patients' concerns [7]; lastly, due to their slowing metabolism and the blood supply shortage [8] of their skin, the time for wound healing was relatively long, and they feared wound dehiscence with a sense of discomfort and pruritus, finally adding some burden, so they were afraid of falling asleep, didn't dare to attend social activities, feared doing housework and were timid and overcautious in daily life.

After 3 months, the patients' mental disorders have greatly been improved. The majority of them got used to pacemakers' working, and the scores increased significantly. In the meantime, their abilities to deal with daily life have been restored gradually, they also participated in community activities more frequently, and their scores have grown to a large degree. 6 months after the operation, their mental disorders were almost gone, their daily life was not affected by the working pacemakers, most of them were willing to participate in social activities and communicate with others, and they were optimistic, showing a positive attitude towards life.

\section{Conclusion}

In conclusion, systematic family nursing guidance can prevent and solve all kinds of problems for patients with implanted pacemakers coming across. It is also proved to be an effective nursing method, and can significantly improve the patients' quality of life with implanted pacemakers. It is remarkable that during nursing guidance, it is necessary to establish the trustfulness to the voices of patients, making explanations to them patiently, observing carefully the patients' behavior and performance and instructing them attentively, which can make the 
elderly patients smoothly get rid of such trouble and return to families and the society. This study is limited to improve the quality of life for the patients, and the research on the improvement of the quality of life for the patients' families still needs to be further carried out.

\section{Conflicts of Interest}

The authors declare no conflicts of interest regarding the publication of this paper.

\section{References}

[1] Chen, K., Ren, Y.-R., Zhang, Y., Chen, X., Zhang, Q., Li, H., Fan, Y.-Q., Xie, Y.-S. and Wang, C.-Q. (2017) Effect and Safety Evaluation of Pacemaker Implatation in Extreme Elderly Patients. Chinese Journal of Cardiac Pacing and Electrophysiology, $1,12-14$

[2] Fang, X.Y., Mao, J.L., Cui, H.S. and Zhao, X.D. (2016) The Effect of Mental State before Implantation on Quality of Life in Patients with Pacemakers. Chinese Journal of Cardiac Pacing and Electrophysiology, 30, 54-55.

[3] Liang, J.J., Yang, B., Wu, S., Huang, H. and Chen, F. (2011) Study on Quality of Life after Implantation of Pacemaker in Aged Patients. Chinese Journal of Cardiovascular Rehabilitation Medicine, 20, 196-197.

[4] Shen, Z.Y., Zhen, F., Zhong, Z.Q., Hu, M.H., Ding, S.Q. and Xiao, Y.C. (2018) A Qualitative Research on the Psychological Experience of Patients after Permanent Pacemaker Implementation. Nursing Journal of Chinese People's Liberation Army, $35,19-24$.

[5] Li, L., Wang, H.M. and Shen, Y. (2002) The Chinese Development of SF-36 Health Survey Scale and Its Performance Testing. Chinese Journal of Preventive Medicine, 36, 109.

[6] Cheng, X., Wang, L. and Xie, H. (2015) Quality of Life of the Elderly at Home and Its Influential Factors. Chinese General Practice, 18, 1769-1770.

[7] Di, C.Y. and Lin, W.H. (2012) Recognition of Managed Ventricular Pacing. Chinese Journal of Cardiac Pacing and Electrophysiology, 26, 168-169.

[8] Shi, S.M., Wu, F.K., Wen, X.H., Wang, B.H. and Liu, Y. (2015) Clinical Study of Hospital Infections after Permanent Pacemaker Implantation in Elderly Patients. Chinese Journal of Nosocomiology, 25, 3266-3267. 